\title{
Modeling and Analysis of SOGI-PLL/FLL-based Synchronization Units: Stability Impacts of Different Frequency-feedback Paths
}

\author{
Zhang, Chen; Foyen, Sjur; Suul, Jon Are; Molinas, Marta
}

Published in:

IEEE Transactions on Energy Conversion

Link to article, DOI:

10.1109/TEC.2020.3041797

Publication date:

2021

Document Version

Peer reviewed version

Link back to DTU Orbit

Citation (APA):

Zhang, C., Foyen, S., Suul, J. A., \& Molinas, M. (2021). Modeling and Analysis of SOGI-PLL/FLL-based Synchronization Units: Stability Impacts of Different Frequency-feedback Paths. IEEE Transactions on Energy Conversion, 36(3), 2047 - 2058. https://doi.org/10.1109/TEC.2020.3041797

\section{General rights}

Copyright and moral rights for the publications made accessible in the public portal are retained by the authors and/or other copyright owners and it is a condition of accessing publications that users recognise and abide by the legal requirements associated with these rights.

- Users may download and print one copy of any publication from the public portal for the purpose of private study or research.

- You may not further distribute the material or use it for any profit-making activity or commercial gain

- You may freely distribute the URL identifying the publication in the public portal 


\title{
Modeling and Analysis of SOGI-PLL/FLL- based Synchronization Units: Stability Impacts of Different Frequency-feedback Paths
}

\author{
Chen Zhang, Sjur Føyen, Jon Are Suul, Member, IEEE, Marta Molinas, Member, IEEE
}

\begin{abstract}
Second-order Generalized Integrator (SOGI)-based quadrature-signal-generator (QSG) together with either a phaselocked-loop (PLL) or a frequency-locked-loop (FLL) constitute two types of typical synchronization units (i.e., SOGI-PLL and FLL) that have been widely used in grid-tied converter systems. This paper will reveal and clarify the stability issue of these two synchronization units arising from different implementations of the frequency-feedback-path (FFP) connecting the SOGI-QSG and the PLL/FLL. In this regard, four types of FFP implementations that are frequently seen in the literature will be discussed. Although different implementations of the FFP will not affect the steady-state frequency adaptation, their dynamical effects on the small-signal stability of SOGI-PLL/FLL remain concealed. To this end, this paper will present a comprehensive stability assessment and comparative analysis of SOGI-PLL/FLL focusing on the FFP issue. To extend the applicability and accuracy of discussions, all the analyses will be fulfilled by using a parameter space-oriented stability assessment method formulated in the linear-time periodic (LTP) framework. The obtained results are verified by time-domain simulations, and the main findings are further interpreted by using appropriate analytical models.
\end{abstract}

Index Terms - FLL, PLL, synchronization, SOGI, stability, LTP, frequency feedback.

\section{INTRODUCTION}

$\mathrm{G}$ RID-SYNCHRONIZATION is one of the most fundamental functions required in controls of grid-connected Voltage Source Converters (VSCs). In three-phase VSCs, grid synchronization is commonly achieved by using synchronousreference-frame (SRF)-based phase-locked-loop (PLL) [1], [2], of which the input is the space vector formed by the three-phase grid voltages. For single-phase VSCs, such a space vector is usually synthesized by a quadrature-signal-generator (QSG), and the Second-order Generalized Integrator (SOGI) [4] based on the principle of GI [5], [6] is widely adopted for this purpose.

The conventional SOGI-QSG can output an ideal orthogonal signal when the applied grid voltage has a fixed-frequency and is equal to the specified resonance frequency of the SOGI. However, many electrical systems such as microgrids and

This work was in part supported by the Norwegian University of Science and Technology (NTNU), and in part supported by the Technical University of Denmark (DTU).

C. Zhang is with the Department of Electrical Engineering, DTU, 2800, Lyngby, Denmark (email: chezh@elektro.dtu.dk ).

M. Molinas are with the Department of Engineering Cybernetics, NTNU, 7034, Trondheim, Norway (email: marta.molinas@ntnu.no). distributed energy systems (DES) experience time-varying line frequencies, e.g., caused by load variations. Under such occasions, the in-phase and quadrature signals generated by the SOGI-QSG with a fixed resonance frequency will be asymmetric [4], affecting the precision and robustness of the grid-synchronization and the control of VSCs.

This sensitivity to frequency variations can be largely alleviated by endowing the SOGI-QSG with the ability of frequency adaptation. To this end, many frequency adaptive laws have been proposed [4]-[10], including techniques known as adaptive-notch-filtering (ANF) [11]. A comprehensive review is performed in [12] and [13]. Among these methods, the following two frequency adaptive SOGI-QSG are most appealing for practical applications due to their simplicity in implementations: the SOGI-PLL [3], [4], and the SOGI-FLL (frequency-locked-loop) [10]. In these two realizations, the frequency adaptation is achieved by adding a frequencyfeedback path (FFP) between the PLL/FLL and the SOGI-QSG, which is illustrated in Fig. 1 (a) (where the dotted line is only used in FLL). Furthermore, as given in Fig. 1 (b)-(e), depending on where the FFP is fed into the SOGI-QSG, four types of FFP implementations can be identified. As examples of common implementations, the Type-I FFP is adopted in [7] and [10], while the Type-II can be found in use in [4] and [12].

The ability of frequency adaption opens many possibilities and opportunities for applying the SOGI-PLL/FLL in achieving the grid-synchronization and converter controls under diverse grid conditions. For instance, a sequence filter [5] can be realized by using a double SOGI (DSOGI) configuration [14], which can be applied to improve the synchronization ability and gain more degree of freedom for converter controls under asymmetric grid conditions. Furthermore, several SOGIs can be composed together to obtain a multi-harmonics extractor [15][16], which can be utilized to improve the power quality of converters under severe grid distortions by further adding harmonic current controllers.

Despite the progress that has been made in the aforementioned aspects of SOGI-QSG, there is a fundamental yet

Sjur Føyen is with the Department of Electric Power Engineering, NTNU, 7034, Trondheim, Norway, email: foyen.sjur@ntnu.no.

J. A. Suul is with SINTEF Energy Research, 7491 Trondheim, Norway and also with the Department of Engineering Cybernetics, NTNU, 7034 Trondheim, Norway (Jon.A.Suul@ sintef.no). 


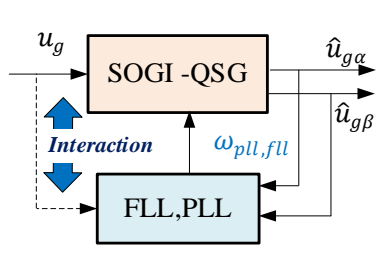

(a)

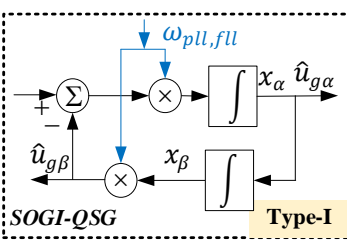

(b)

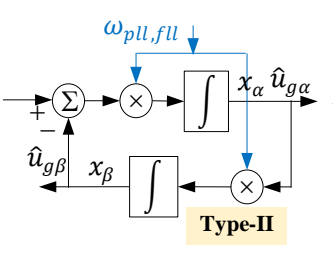

(c)

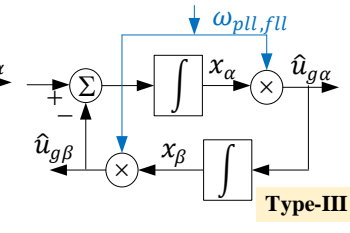

(d)

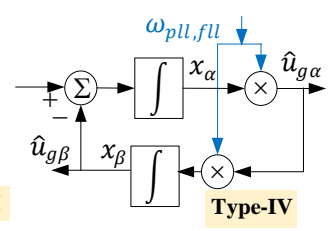

(e)

Fig. 1 (a) a feedback representation of SOGI-PLL/FLL; (b)-(e) four types of FFP implementations

significant issue that remains concealed, which is about the FFP implementation and its dynamic impacts. By analyzing the configurations given in Fig. 1 (b)-(e) it can be obtained that the choice of different FFP implementations will not affect the steady-state characteristics of SOGI-QSG (i.e., the efficacy of steady-state frequency adaptation). However, since the FFP introduces nonlinearities to the synchronization units, its dynamical impacts on SOGI-PLL/FLL are anticipated to exist. In which, an in-depth study and clarification on their smallsignal dynamics and stability would be necessary, which is also the first step toward more sophisticated analyses (e.g., the largesignal stability analysis). To the authors' best knowledge, such an analysis is lacking in the literature.

To perform the small-signal analysis, system modeling is an essential step. In which, the modeling of SOGI-PLL/FLL is usually conducted with the help of the averaging technique so that the time-variant effects of the system can be removed [12], which in turn leads to a simpler modeling process. Another assumption commonly made for achieving a quasi-steady-state model of a SOGI-QSG is that the dynamics of the SOGI-QSG are assumed to be much faster than those of the FLL frequency tracking [9], [15]. Based on these assumptions, a first-order model of SOGI-FLL has been developed in [15]. Undeniably, such simplified models can gain insights into the behavior of SOGI-FLL, however, they are not suitable for rigorous and quantitative stability assessment due to ignored effects (e.g., the time-variance). To arrive at a model that is sufficiently rigorous for small-signal stability analysis, the linear-time-periodic (LTP) method [17] can be applied to better cope with the timevariance issue [18], [19]. Due to this merit, in this paper, the modeling and analysis of SOGI-PLL/FLL will be performed in the LTP framework.

Overall, this paper will contribute to the following two aspects: 1) reveal and clarify the small-signal stability impacts of different FFP implementations for SOGI-PLL/FLL; 2) compare and discuss the stability performance of SOGI-PLL and -FLL over wide parameter space. The remainder of this paper is organized as follows:

Section II introduces the parameter space-oriented stability assessment method. Based on this method, Section III performs a comprehensive stability assessment of SOGI-PLL/FLL under different FFP implementations. To consolidate the understanding of the main findings obtained from the numeric analysis, interpretations of those main findings will be provided in Section IV. Finally, Section V draws the main conclusions.

\section{Parameter SPaCe-ORIENTEd Modeling AND ANAlysis METHOD FOR SOGI-PLL/FLL}

This section will first introduce some fundamentals of the LTP method; afterward, the parametric stability assessment method and the approach of unifying the parameter space of SOGI-PLL/FLL will be elaborated.

\section{A. The LTP method}

The SOGI-PLL/FLL are essentially time-varying systems as the outputs of SOGI-QSG are periodic signals in steady-state. When modeling and analyzing systems with periodic steadystate (PSS), the LTP method is usually applied. In which, the LTP system (2) with a period of $T$ can be obtained by linearizing the system (1) around its PSS conditions.

$$
\begin{aligned}
\dot{\boldsymbol{x}} & =\boldsymbol{f}(t, \boldsymbol{x}, \boldsymbol{u}) \\
\boldsymbol{y} & =\boldsymbol{g}(t, \boldsymbol{x}, \boldsymbol{u}) \\
\Delta \dot{\boldsymbol{x}} & =\boldsymbol{A}(t) \Delta \boldsymbol{x}+\boldsymbol{B}(t) \Delta \boldsymbol{u} \\
\Delta \boldsymbol{y} & =\boldsymbol{C}(t) \Delta \boldsymbol{x}+\boldsymbol{D}(t) \Delta \boldsymbol{u}
\end{aligned}
$$

In (2), $\boldsymbol{A}(t), \boldsymbol{B}(t)$ and $\boldsymbol{C}(t), \boldsymbol{D}(t)$ are partial differentials of $\boldsymbol{f}$ and $\boldsymbol{g}$ and they are functions with a period of $T$.

Unlike the stability analysis of linear-time-invariant (LTI) systems, stability of the LTP system cannot be assessed by directly calculating the eigenvalues of $\boldsymbol{A}(t)$. To address this issue, (2) can be transformed into a state-space-like model, where the states, inputs, and outputs are all represented by corresponding Fourier coefficients. Such a model is referred to as the harmonic state space (HSS) model (please see [17] for details) written as:

$$
\begin{aligned}
& \dot{\mathcal{X}}=(\mathcal{A}-\mathcal{N}) \mathcal{X}+\mathcal{B} \cdot \mathcal{U} \\
& \mathcal{Y}=\mathcal{C} \cdot \mathcal{X}+\mathcal{D} \cdot \mathcal{U}
\end{aligned}
$$

where the states $\mathcal{X}$ and inputs $\mathcal{U}$ are the Fourier coefficients of $\Delta \boldsymbol{x}$ and $\Delta \boldsymbol{u}$; while $\mathcal{A}, \mathcal{B}, \mathcal{C}, \mathcal{D}$ are Toeplitz format matrices whose entries are Fourier coefficients of $\boldsymbol{A}(t), \boldsymbol{B}(t), \boldsymbol{C}(t), \boldsymbol{D}(t)$ respectively; $\mathcal{N}$ is a block diagonal matrix consisting of $\mathrm{j} k \omega_{1} \boldsymbol{I}, k=0, \pm 1, \ldots \pm N$.

It is noticed that the harmonic-order $N$ has to be defined before (3) can be solved numerically. Usually, $N$ is chosen according to the number of dominant harmonics existing in the system. In this paper, $N=4$ is selected because the frequency responses of SOGI-PLL/FLL above this order are overall small (please see Appendix-A). Once $N$ is defined, small-signal stability of (2) can be equivalently studied by the truncated model of (3), i.e., the eigenvalues of $\mathcal{A}-\mathcal{N}$ [20]. 


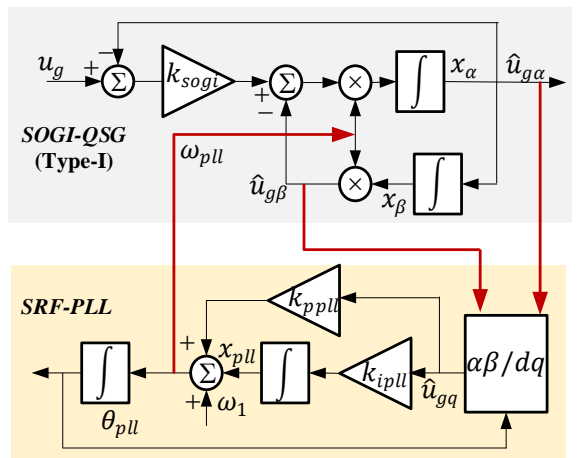

(a)

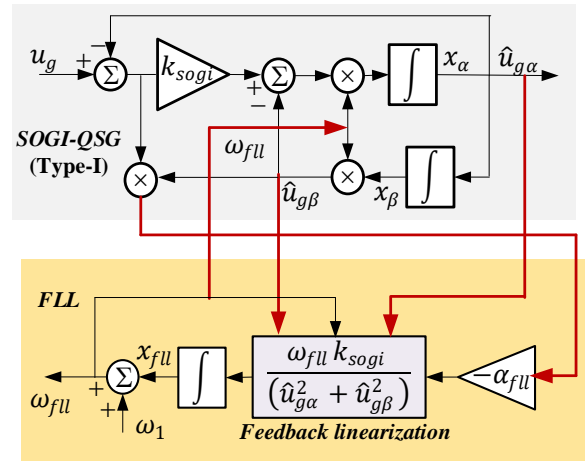

(b)

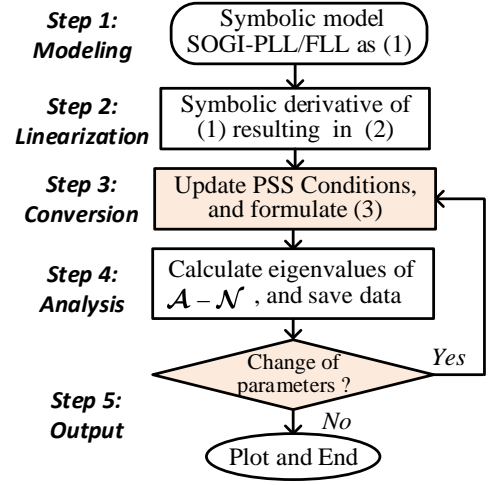

(c)

Fig. 2. (a) SOGI-PL with Type-I implementation. (b) SOGI -FLL with Type-I implementation. Implementations of (a) and (b) are also used in [10]; SOGIPLL/FLL with other types of FFP implementations (given in Fig. 1) can be drawn similarly as (a) and (b). (c) The flow chart for the parameter space-oriented modeling and analysis method applied to SOGI-PLL/FLL.

\section{B. A parameter space-oriented modeling and analysis method for SOGI-PLL/FLL}

Modeling and analysis of SOGI-PLL/FLL using the abovementioned LTP method primarily follow three steps:

1) Establish the state-space model;

2) Perform linearization around the PSS conditions;

3) Transform the linearized model into HSS for analysis. Implementation of the above steps using analytic methods is feasible for individual cases, however, the procedure becomes extremely cumbersome if the parametric study is aimed at. For instance, the PSS conditions in step 2) should be re-evaluated (which are typically obtained from lengthy time-domain simulations) and inserted in the analytic model. This process will be repeated for every change of the system's parameters.

Therefore, to arrive at an efficient method for parametric studies, the above procedures will be automatically fulfilled in MATLAB using symbolic and numeric calculations in this paper. To illustrate this, next, the SOGI-PLL/FLL with Type-I SOGI-QSG will be analyzed as an example.

\section{1) State-space modeling of SOGI-PLL/FLL (Type-I)}

According to Fig. 2 (a), the state-space model of the SOGIPLL can be described by the following equations:

$$
\left\{\begin{array}{l}
\dot{x}_{\alpha}=k_{\text {sogi }}\left(u_{g}-x_{\alpha}\right) \omega_{p l l}-\omega_{p l l}^{2} x_{\beta} \\
\dot{x}_{\beta}=x_{\alpha} \\
\dot{x}_{p l l}=k_{i p l l} \hat{u}_{g q} \\
\dot{\delta}_{p l l}=k_{p p l l} \hat{u}_{g q}+x_{p l l}
\end{array}\right.
$$

and,

$$
\left\{\begin{array}{l}
\hat{u}_{g q}=-\sin \left(\omega_{1} t+\delta_{p l l}\right) x_{\alpha}+\cos \left(\omega_{1} t+\delta_{p l l}\right) \omega_{p l l} x_{\beta} \\
\omega_{p l l}=x_{p l l}+\omega_{1}+k_{p p l l} \hat{u}_{g q}
\end{array}\right.
$$

Similarly, according to Fig. 2 (b), the state-space model of SOGI-FLL can be described by the following equations:

$$
\left\{\begin{array}{l}
\dot{x}_{\alpha}=k_{\text {sogi }}\left(u_{g}-x_{\alpha}\right) \omega_{f l l}-\omega_{f l}^{2} x_{\beta} \\
\dot{x}_{\beta}=x_{\alpha} \\
\dot{x}_{f l l}=\left(u_{g}-x_{\alpha}\right) \omega_{f l} x_{\beta} k_{f f}
\end{array}\right.
$$

where

$$
\left\{\begin{array}{l}
k_{f f}=-\alpha_{F L L} \frac{\omega_{f l l} k_{s o g i}}{x_{\alpha}^{2}+\omega_{f l l}^{2} x_{\beta}^{2}} \\
\omega_{f l l}=\omega_{1}+x_{f l l}
\end{array}\right.
$$

Then, (4)-(7) can be formulated in MATLAB using the command sym/syms for later symbolic calculations.

\section{2) Linearization}

Linearization of (4)-(7) can be fulfilled analytically, however, since this paper aims at a systematic analysis of stability, the linearization process is assisted with using the symbolic calculation of MATLAB, i.e., the command jacobian ().

\section{3) Convert the linearized model into HSS}

When converting (2) into (3), it is equivalent to transform $\boldsymbol{A}(t), \boldsymbol{B}(t), \boldsymbol{C}(t), \boldsymbol{D}(t)$ into the Toeplitz matrices $\mathcal{A}, \mathcal{B}, \mathcal{C}, \mathcal{D}$. In which, the Fourier coefficients of $\boldsymbol{A}(t), \boldsymbol{B}(t), \boldsymbol{C}(t), \boldsymbol{D}(t)$ for formulating $\mathcal{A}, \mathcal{B}, \mathcal{C}, \mathcal{D}$ should be obtained, which can be fulfilled by using the command $F F T()$ in MATLAB.

\section{4) Update the PSS conditions}

Notably, extracting the Fourier coefficients of e.g., $\boldsymbol{A}(t)$ for obtaining $\mathcal{A}$ should be conducted upon an established steadystate, i.e., the PSS conditions should be known beforehand. In this regard, a Newton-based iterative method for fast PSS extraction is developed in [23]. The method will be adopted in this paper as the iterative procedure is self-contained and can be readily integrated into later parametric stability analysis.

Once $\mathcal{A}, \mathcal{B}, \mathcal{C}, \mathcal{D}$ are obtained according to the updated PSS conditions, the small-signal stability under various parameters can be readily assessed by calculating the eigenvalues of $\mathcal{A}-\mathcal{N}$. A flow chart of the above-illustrated parameter spaceoriented modeling and analysis method is given in Fig. 2 (c).

After the above preparations, one more step is required for achieving a fair and straightforward stability comparison of SOGI-PLL and -FLL, which is discussed in the next.

\section{Parameter unification of FLL and PLL in terms of the settling time}

From Fig. 2 (a) and (b) it can be clearly seen that SOGIPLL/FLL have the same SOGI-QSG if the FFP implementation 
and $k_{\text {sogi }}$ are the same. Therefore, the focus of parameter unification is placed on subsystems of FLL and PLL.

First, for the FLL given in Fig. 2 (b), it has been shown in [10] that it can be approximated by a first-order system (which is given in Fig. 3 (a)) due to the applied feedback linearization. Furthermore, the gain $\alpha_{F L L}$ of the first-order model is reciprocal to the time constant, which characterizes the settling time of FLL (e.g., $\left.t_{s t} \approx 4 / \alpha_{F L L}\right)$. Therefore, $\left(\alpha_{F L L}, k_{s o g i}\right)$ forms a parameter space for the SOGI-FLL.

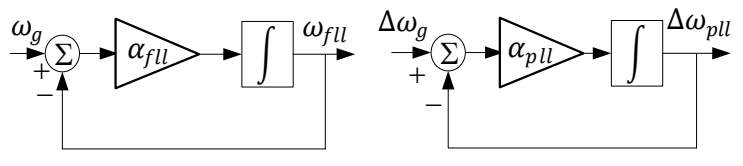

(a) FLL first-order approximation

(b) PLL first-order approximation

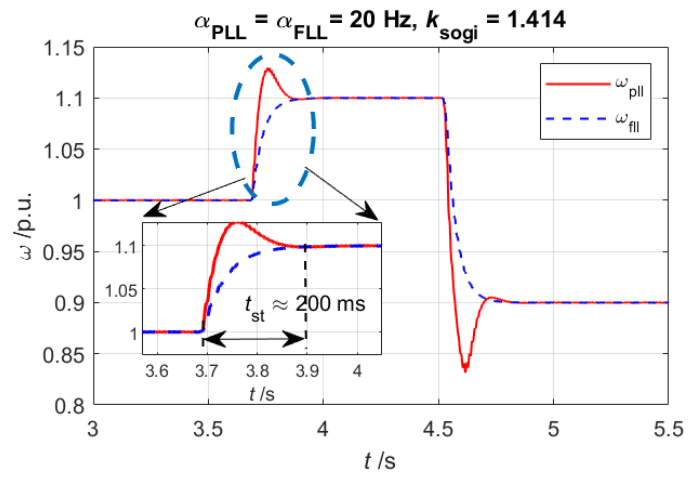

(c) A comparison of time-domain responses

Fig. 3 First order approximation of the FLL and PLL

Next, to arrive at a similar parameter space for the SOGIPLL, the main objective is to find a parameter that resembles $\alpha_{F L L}$, i.e., characterize the settling time of PLL at least in a small-signal sense (as this paper focuses on small-signal issues). To this end, the following analysis is performed.

First, the linearized PLL model can be obtained as [22]

$$
\Delta \theta_{p l l}=\underbrace{\frac{k_{p l l} s+k_{i p l l}}{s^{2}+\left(k_{p l l} s+k_{i p l l}\right) U_{0}}}_{G_{\mathrm{pll} \_\theta}(s)} \Delta \hat{u}_{g q}
$$

for small-signal approximation, this equation $\Delta \hat{\theta}_{g}=\Delta \hat{u}_{g q} / U_{0}$ can be used, where $U_{0}$ is the nominal and peak value of grid voltage. Then, multiplying both sides of (8) with the Laplace variable $s$, the following equation can be obtained:

$$
\underbrace{s \Delta \theta_{p l l}}_{\Delta \omega_{p l l}}=\frac{\left(k_{p l l} s+k_{i p l l}\right) U_{0}}{s^{2}+\left(k_{p l l} s+k_{i p l l}\right) U_{0}} \cdot s \Delta \theta_{g}
$$

Based on (9), if the design: $k_{p p l l}=\frac{2 \alpha_{P L L}}{U_{0}}, k_{i p l l}=\frac{2 \alpha_{P L L}^{2}}{U_{0}}$ is applied, then (9) is simplified into

$$
\Delta \omega_{p l l}=\frac{2 \alpha_{P L L} s+2 \alpha_{P L L}^{2}}{s^{2}+2 \alpha_{P L L} s+2 \alpha_{P L L}^{2}} \Delta \omega_{g}
$$

It can be seen that the introduced gain $\alpha_{P L L}$ represents the decaying factor (i.e., inverse of time constant) of the secondorder system of PLL, which implies that the PLL can be approximated by a first-order system if the focus is on the settling time. Then, comparing Fig. 3 (a) and (b) it can be readily obtained that $\alpha_{P L L}$ has the same meaning as $\alpha_{F L L}$, i.e., depicting the system's setting time.

Based on the above analysis, the parameter unification of SOGI-PLL and -FLL is achieved and the resulting twodimensional parameter space is: $\left(\alpha_{P L L / F L L}, k_{\text {sogi }}\right)$. To further show the feasibility of this unification, a time-domain response comparison of SOGI-FLL and -PLL subjected to a step change of grid-frequency is applied, the results are shown in Fig. 3 (c). It can be seen that under the same parameter space, the simulated settling time of SOGI-PLL is similar to that of SOGIFLL, which is also close to the estimated one using $t_{s t}=4 / \alpha_{P L L / F L L}=0.2 \mathrm{~s}$. Therefore, the presented parameter unification method is feasible.

\section{PARAMETRIC STABILITY ASSESSMENTS OF SOGI- PLL/FLL UNDER DIFFERENT FFP IMPLEMENTATIONS}

In this section, the comparative stability assessment of SOGIPLL/FLL under different FFP implementations will be conducted over the unified parameter space $\left(k_{\text {sogi }}, \alpha_{P L L / F L L}\right)$. This can be fulfilled by repeatedly calling the method in Fig. 2 (c). Before proceeding with this analysis, the eigenvalues of an LTP system will be shortly discussed to acquire a better understanding of forthcoming stability plots.

\section{A. An example of calculated eigenvalues}

As illustrated in Fig. 4, eigenvalues calculated from the algorithm in Fig. 2 (c) contain frequency-shifted copies. The occurrence of this phenomenon is attributed to the inherent properties of LTP systems [20]. However, in terms of stability judgments, only the real parts of the weakest modes (as highlighted in Fig. 4) are most relevant for analysis. Moreover, since the real parts of these shifted modes are identical, they can be represented by a unified variable, e.g., $\operatorname{Re}[\lambda]$.

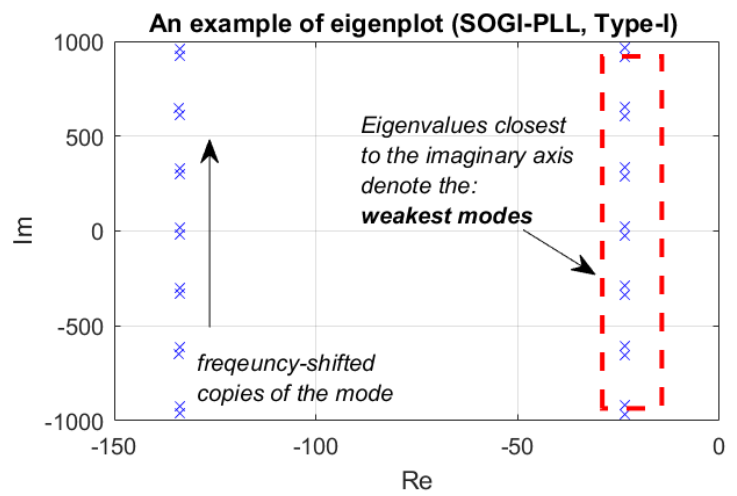

Fig. 4 An example of eigenvalue plot under SOGI-PLL with Type-I implementation $\left(\alpha_{P L L}=20 \mathrm{~Hz}, k_{\text {sogi }}=1\right)$

Based on this notation, generally, $\operatorname{Re}[\lambda]>0$ indicates an unstable system, otherwise, the system is stable. Besides, $R e[\lambda]$ also reflects the system's stability margin, e.g., the system will be more stable if the value of $\operatorname{Re}[\lambda]$ is more negative. Based on these considerations, in the following analysis, $\operatorname{Re}[\lambda]$ will be used as the indicator for parametric stability assessment. 

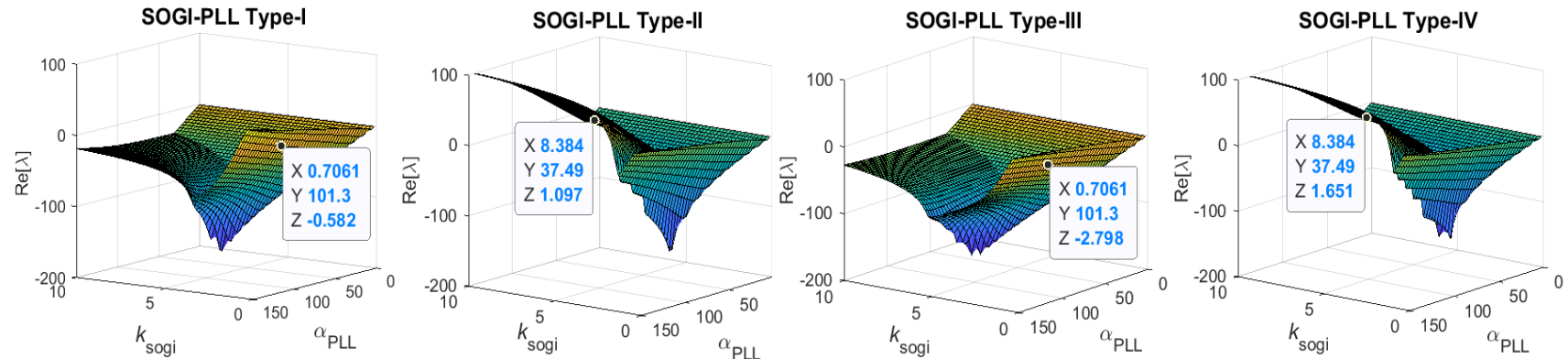

(a) Stability traits of SOGI-PLL under different FFP implementations
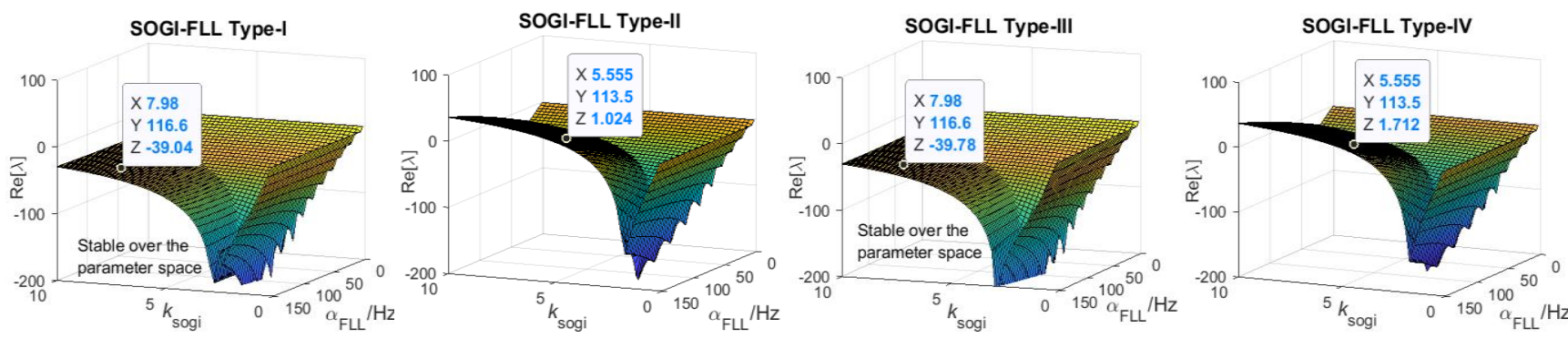

(b) Stability traits of SOGI-FLL under different FFP implementations

Fig. 5 Parametric stability assessments for SOGI-PLL and SOGI-FLL under all the four types of FFP implementations ( $x$ - $y$ plane denotes the parameter space $\left(k_{\text {sogi }}, \alpha_{P L L / F L L}\right)$; while the $z$-axis denotes the real part of the weakest modes (i.e., $\operatorname{Re}[\lambda]$ ).

\section{B. Stability trait analysis of SOGI-PLL/FLL under different FFP implementations}

According to the algorithm in Fig. 2 (c), three-dimensional plots characterizing the parametric stability traits of SOGIPLL/FLL under different FFP implementations can be obtained, as shown in Fig. 5. In these plots, the $x-y$ plane denotes the parameter space $\left(k_{s o g i}, \alpha_{P L L / F L L}\right)$; while the $z$-axis denotes the value of $\operatorname{Re}[\lambda]$.

\section{1) Stability trait analysis of SOGI-PLL}

First, according to Fig. 5 (a), a qualitative understanding of how parameter variations will affect the stability of SOGI-PLL can be immediately obtained. For example, see the plot of TypeI SOGI-PLL and assume that $k_{\text {sogi }}$ is fixed around 10 . Then by inspecting the value of $\operatorname{Re}[\lambda]$ along the $\alpha_{P L L}$-axis, it can be found that the increase of $\alpha_{P L L}$ will first lead to a decrease in $\operatorname{Re}[\lambda]$, then followed by a slightly increase. This means under this type of parameter variation, stability of the SOGI-PLL will be first enhanced then slightly deteriorated.

Apart from the qualitative analysis, a quantitative analysis of stability can also be achieved by examining the value of $\operatorname{Re}[\lambda]$ (e.g., see the data specified on the surfaces of those plots). From the value of $\operatorname{Re}[\lambda]$, not only stability conclusion can be drawn (i.e., stable or not) but also the information of stability margin can be obtained. This delicate analysis will be discussed in Section III-D, along with time-domain simulations.

Next, by observing the overall stability traits of SOGI-PLL in Fig. 5 (a), the following findings can be obtained:

1) Type-I and -III can be classified as one group because they exhibit a similar stability trait (i.e., Group I in Fig. 6).

2) Type-II and -IV belong to another group as they share another similar stability trait (i.e., Group II in Fig. 6).
3) By further observing the configurations of FFPs under each group, it can be found that the stability trait is more sensitive to the FFP location in the quadrature path than in the in-phase path.

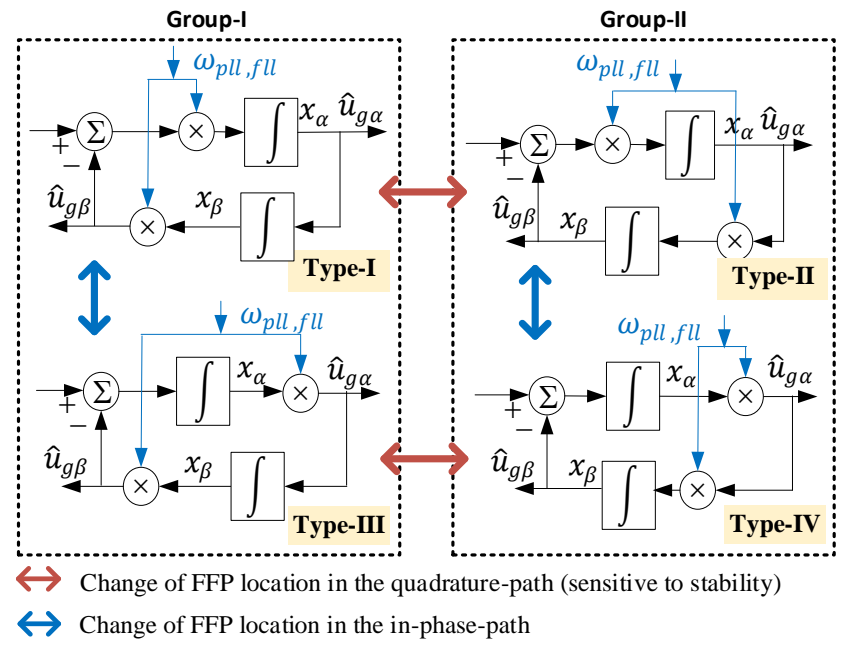

Fig. 6 Classification of FFP implementations according to their stability traits (i.e., Type-I and -III has a similar stability trait, while Type-II and -IV exhibit another similar stability trait, this plot applies to both SOGI-PLL and -FLL)

\section{2) Stability trait analysis of SOGI-FLL}

Stability trait plots of SOGI-FLL under four types of FFP implementations are shown in Fig. 5 (b). Following a similar analysis as the SOGI-PLL, it can be readily confirmed that the above-listed conclusions are all applicable to the SOGI-FLL, thus they are not repeated here. 

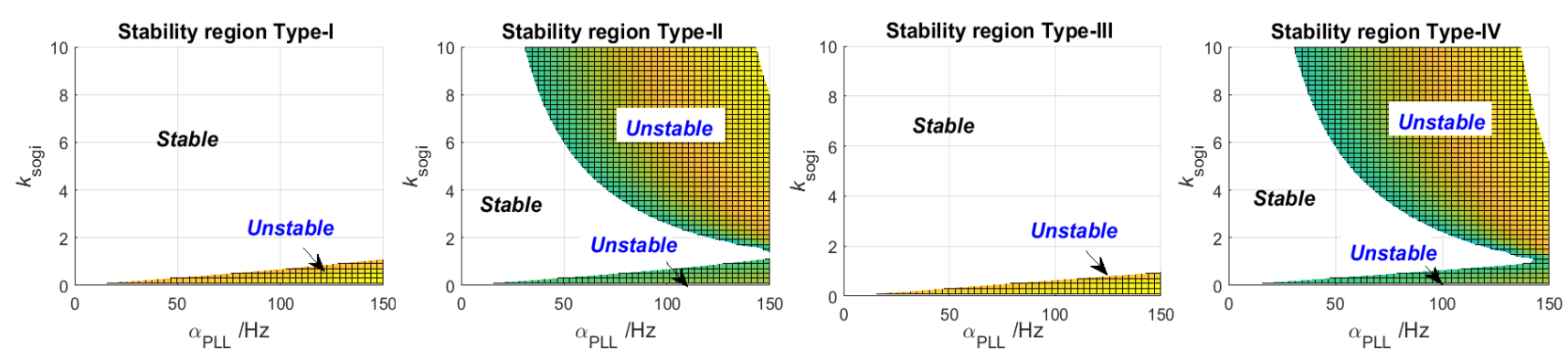

(a) stability regions of SOGI-PLL under different FFP implementations
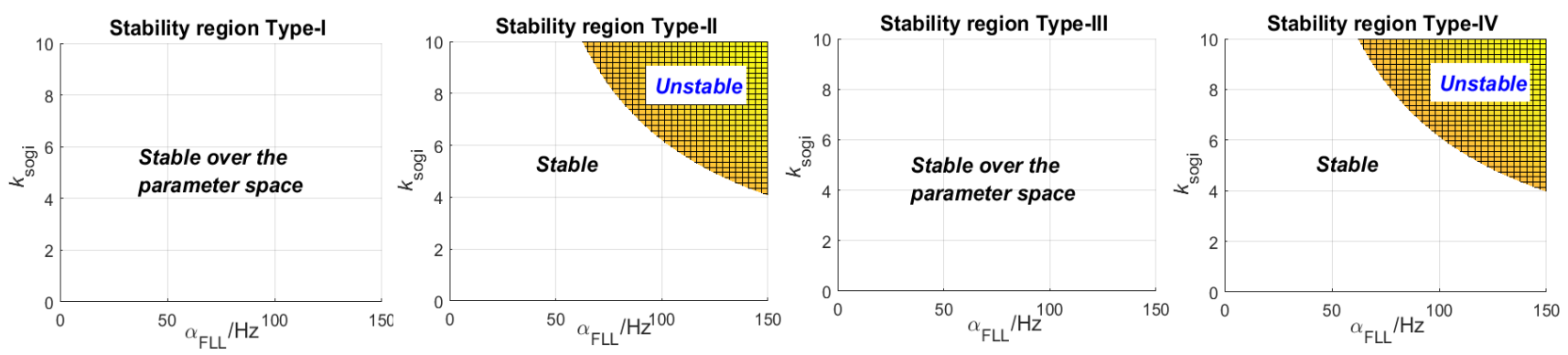

(b) stability regions of SOGI-FLL under different FFP implementations

Fig. 7 Stability region comparisons of SOGI-PLL and SOGI-FLL under different FFP implementations (shaded area denotes unstable region while the blank area denotes the stable region over the prescribed parameter space)

\section{Stability performance analysis of SOGI-PLL/FLL under different FFP implementations}

Although from Fig. 5 one could readily acquire a full picture of the stability trait over the parameter space, it is not easy to further identify which FFP implementation or which type of synchronization unit is better with respect to the stability performance. To this end, in this section, another form of presentation will be used, referred to as the stability region plot. The idea is to delimit a prescribed parameter space into stable and unstable zones. Afterward, the system with a larger stable zone is then considered to have a better stability performance.

According to the above description, the stability region plot can be conveniently found by projecting the stability trait plot onto the plane $z=0$ (i.e., where $\operatorname{Re}[\lambda]=0$ ). Specifically, projecting the space above $z=0$ (i.e. where $\operatorname{Re}[\lambda]>0$ ) will result in the "unstable" region while projecting the space below $z=0$ will obtain the "stable" region. Following this procedure, Fig. 5 can be converted into stability region plots as shown in Fig. 7. Based on these plots, the afore-raised questions can be readily resolved, which are shown below:

1) First, overall, it can be obtained that the Type-I/III have a larger stable region than Type-II/IV regardless of the types of synchronization units used. In particular, for SOGI-FLL with Type-I/III, the stable zone covers the entire prescribed parameter space, indicating a superior stability performance.

2) By comparing SOGI-PLL and -FLL under each FFP implementation it can be further obtained that SOGI-FLL has overall better stability performance than SOGI-PLL due to the wider area of the stable region.

3) The above two main conclusions suggest that it is better to configure or implement the SOGI-QSG as Type-I/III to gain more benefits on stability. This conclusion applies to both SOGI-PLL and -FLL.
Based on the stability region plots it can also be observed that the previously identified sensitivity issue is more evident when the SOGI gain $k_{\text {sogi }}$ is large (e.g., see Fig. 7 (a), where the unstable region of Type-II is significantly different from TypeI when $k_{\text {sogi }}$ is large). More discussion on this observation will be given in Section IV. For a convenient reference, the above conclusions are summarized in Table I.

Table I A summary of comparative stability analysis of SOGI-PLL/FLL

\begin{tabular}{l|c|c|c}
\hline \hline \multirow{2}{*}{ FFP type } & \multicolumn{2}{|c|}{ Stability region } & \multirow{2}{*}{ Stability performance $^{2}$} \\
\cline { 2 - 3 } & SOGI-PLL & SOGI-FLL & \\
\hline Type-I & Large ${ }^{1}$ & Entire space & FLL>PLL \\
\hline Type-III & Small & Large & FLL>PLL \\
\hline Type-IV & $\begin{array}{c}\text { Similar to } \\
\text { Type-I }\end{array}$ & $\begin{array}{c}\text { Similar to } \\
\text { Type-I }\end{array}$ & FLL>PLL \\
\hline $\begin{array}{l}\text { Other } \\
\text { conclusions } \\
\text { Type-II }\end{array}$ & $\begin{array}{l}\text { Similar to } \\
\text { Type-II }\end{array}$ & $\begin{array}{l}\text { Type-I/III is overall better than Type-II/IV in } \\
\text { stability performance; } 2) \text { Sensitivity of stability to the }\end{array}$ \\
& $\begin{array}{l}\text { FFP location change is more evident when the SOGI } \\
\text { gain }\left(k_{\text {sogi }}\right) \text { is large. }\end{array}$ \\
\hline
\end{tabular}

On the other hand, the above stability region-based analysis also demonstrates that the parameter space-oriented method using the stability region representation can serve as a useful tool for fast stability tests and parameter designs of SOGIPLL/FLL. For example, parameter designs and optimizations of SOGI-PLL/FLL could be performed within the stable zone of the parameter space so that the small-signal instability would be largely avoided. 


\section{Stability margin analysis and time-domain verifications}

\section{under different FFP implementations}

As mentioned earlier, a quantitative analysis of the system stability margin can be fulfilled by examining how close the value of $\operatorname{Re}[\lambda]$ is to the zero. In the following, this quantitative analysis will be conducted by showing the subtle difference of stability margin existing between either Type-I- and III or Type-II- and IV (although, qualitatively, they have been shown to have a similar stability trait/region in the above analysis).

To fulfill this quantitative analysis, a set of data has been specified on the plots in Fig. 5. For convenient references, they are collected and shown in Table II.

Table II Data set in Fig. 5 and the theoretical stability results

\begin{tabular}{c|c|c|c|c}
\hline \hline & \multicolumn{2}{|c|}{ SOGI-PLL } & \multicolumn{2}{c}{ SOGI-FLL } \\
\cline { 2 - 5 } & $\left(k_{\text {sogi }}, \alpha_{P L L}\right)$ & $\operatorname{Re}[\lambda]$ & $\left(k_{\text {sogi }}, \alpha_{F L L}\right)$ & $\operatorname{Re}[\lambda]$ \\
\hline Type-I & $(0.706,101.3)$ & -0.582 & $(7.98,116.6)$ & -39.04 \\
& & Stable & & Stable \\
\hline Type-II & $(8.384,37.5)$ & 1.097 & $(5.555,113.5)$ & 1.024 \\
& & Unstable & & Unstable \\
\hline Type-III & Same as & -2.798 & Same as & -39.78 \\
& Type-I & Stable & Type-I & Stable \\
\hline Type-IV & Same as & 1.651 & Same as & 1.712 \\
& Type-II & Unstable & Type-II & Unstable \\
\hline \hline
\end{tabular}

From this table, it can be clearly seen that there exists a subtle difference in stability margin between Type-I and -III (or TypeII and -IV) under the same parameter set. For example, the values of $\operatorname{Re}[\lambda]$ for Type-I and -III (SOGI-PLL) are -0.582 and -2.798 respectively, showing that Type-I has a lower stability margin than Type-III. Therefore, in time-domain response, it is anticipated that the SOGI-PLL under Type-I implementation will present a longer settling time than Type-III. This will be shown later. It should be noted that this subtle difference in stability margin will not lead to evident impacts on overall parametric stability performance (since, as already shown, Type-I and -III have a similar size of the stable region).

Before validating the accuracy of the numeric results given in Table II, it can be observed that the values of $\operatorname{Re}[\lambda]$ are chosen intentionally, aiming at a more comprehensive validation. For example, the values of $\operatorname{Re}[\lambda]$ under Type-I/III are chosen to be negative for validating the case of a stable system, while those under Type-II/IV are chosen to be positive for validating the case of an unstable system. Based on this arrangement, time-domain simulations for both SOGI-PLL and -FLL under all the four types of FFP implementations are conducted in PSCAD/EMTDC using the parameters given in Table II. The results are shown in Fig. 8.

According to Fig. 8, it can be seen that for both SOGI-PLL and -FLL, Type-I and-III exhibit a stable system, while Type-II and -IV present an unstable system. These simulated results are consistent with the theoretically predicted stability results given in Table II. Furthermore, when inspecting closer the stable waveforms of SOGI-PLL under Type-I and -III in Fig. 8 (a), it can be seen that indeed the Type-I takes a longer time to settle down at the steady-state compared to Type-III (also the

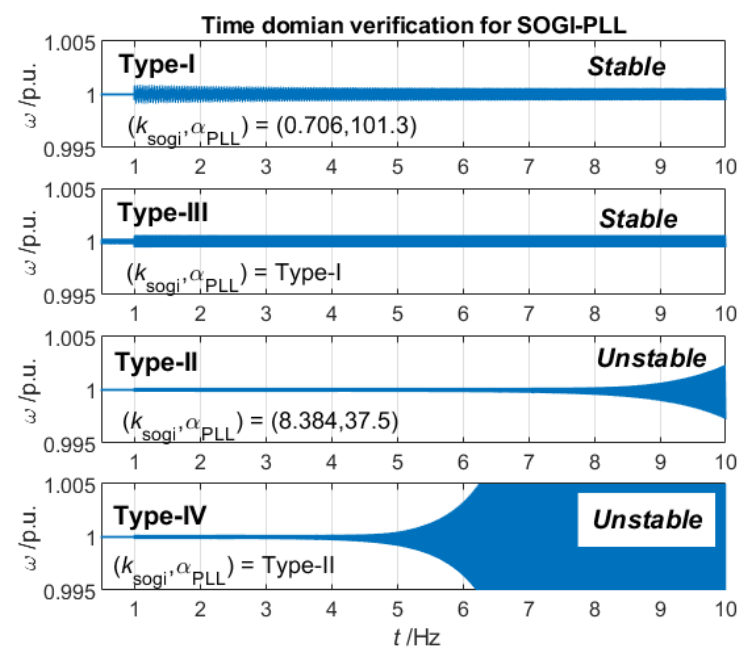

(a) SOGI-PLL

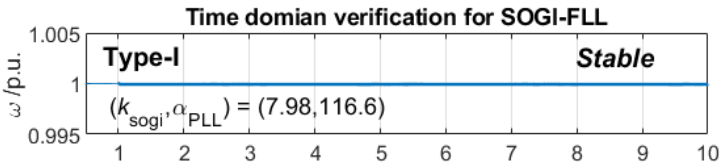

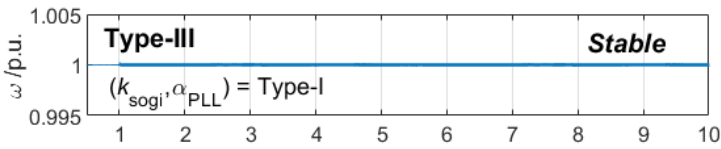

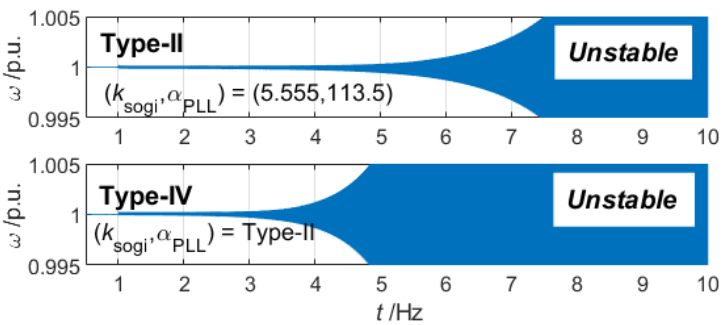

(b) SOGI-FLL

Fig. 8 Time-domain simulations for both SOGI-PLL and -FLL under the four types of FFP implementations (first, the system is initialized with a different set of parameters to ensure a stable operating condition; then around 1s, the parameters are set to the values specified in Table II).

transient of Type-III is smaller). This observation is consistent with the analysis based on the values of $\operatorname{Re}[\lambda]$ in Table II.

Based on a similar analysis of $\operatorname{Re}[\lambda]$, other waveforms can be explained as well. For instance, in Fig. 8 (b), the timedomain responses of SOGI-FLL under Type-I and -III are similar because their values of $\operatorname{Re}[\lambda]$ are very close (given in Table II). Whereas for the unstable waveforms in Fig. 8 (either SOGI-PLL or -FLL), it can be seen that the magnitudes of unstable waveforms under Type-IV grow faster than those under Type-II. This is mainly because the value of $\operatorname{Re}[\lambda]$ for either SOGI-PLL or -FLL under Type-IV is larger than that under Type-II (see the values in Table II).

Overall, the above analysis demonstrates that the theoretic stability results (i.e., the numeric values in the generated stability plots) are valid and accurate. 

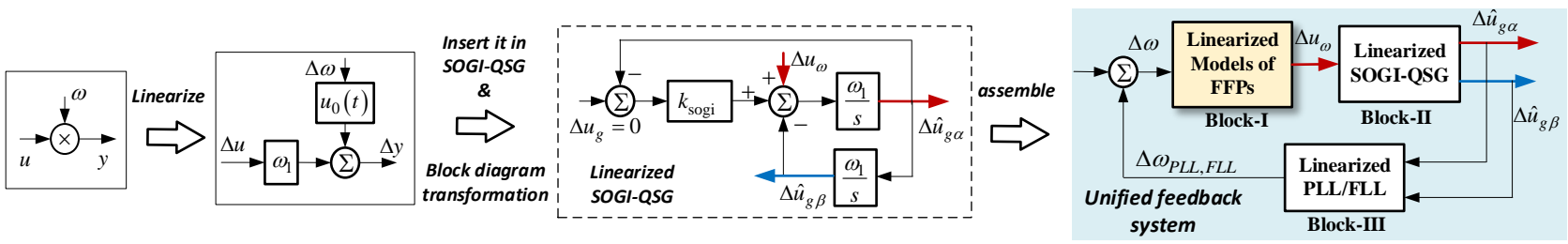

(a) Derivation of the unified feedback system for SOGI-PLL/FLL under different FFP implementations

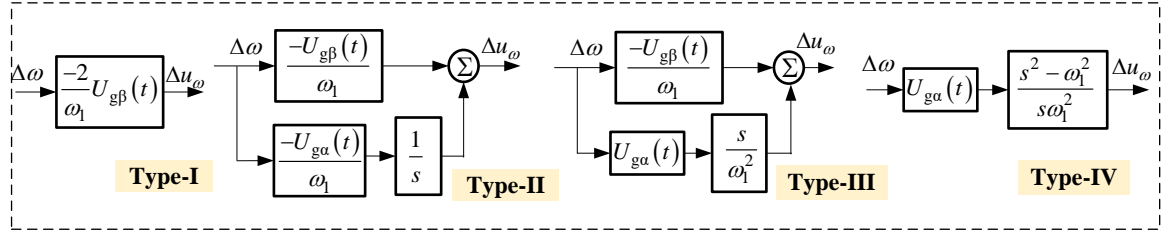

(b) Linearized models of FFPs

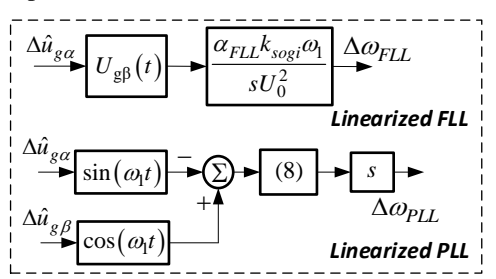

(c) Linearized PLL and FLL

Fig. 9 Linearized control blocks of SOGI-PLL/FLL and the unified feedback system (Block-II is LTI while Block-I and -III are LTP systems)

\section{DISCUSSIONS}

To consolidate the understanding of the conclusions drawn from the numeric stability analysis, this section will provide some interpretations of the main findings from the control system perspective. To this end, a feedback system uniformly characterizing SOGI-PLL/FLL will be first developed.

\section{A. The unified feedback system}

Since this analysis will be concentrated on the impacts of FFP implementations, the frequency variation will be regarded as the main source of perturbation, while the input grid voltage is assumed to be ideal. Then, based on the linearization process given on the left side of Fig. 9 (a), a unified feedback system can be obtained, which is shown on the right side of the figure.

From Fig. 9 (a), it can be seen that the feedback system consists of three blocks, i.e., Block-I III. The model of BlockII is shown in the middle of Fig. 9 (a), while the models of Block-I and -III are given in Fig. 9 (b) and (c). Also, it is noted that Block-II is LTI while Block-I and -III are LTP variant. The presence of LTP blocks indicates that when transforming the overall system into the frequency-domain, frequency couplings will be present (see an example of this effect in Appendix-A). This will result in a high-dimensional model that is difficult to analyze. Therefore, to provide more insights into the system, a simplified frequency-domain model of the unified feedback system focusing on the input and output relation of the same frequency will be developed.

\section{B. The simplified model of the unified feedback system}

Derivation of the simplified frequency-domain model can be generally achieved by merely modeling the frequency components of interest, e.g., in this study, the frequency components at $\omega_{\mathrm{p}} \pm 2 \omega_{1}$ or higher are neglected from the modeling, where $\omega_{\mathrm{p}}$ is the perturbation frequency. Based on this assumption, a simplified control diagram of the unified feedback system can be drawn in Fig. 10. In this figure, for brevity, only the dimensions and types of Block-I III are shown, while their detailed models are given in Appendix-B.

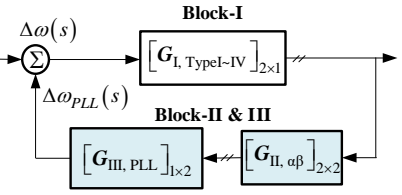

(a) SOGI-PLL

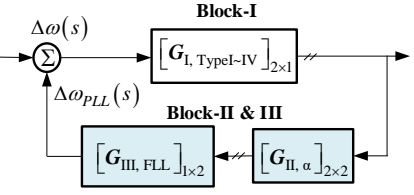

(b) SOGI-FLL
Fig. 10 Simplified model of the unified feedback system

Although this model is greatly reduced from the one used in parametric stability analysis, they are still rather difficult to interpret as the transfer-functions are in fact matrices. To arrive at a scalar index for better interpreting the effects, the below sensitivity function will be used (the negative sign appearing in (11) is due to the systems in Fig. 10 are drawn as positive feedback connections):

$$
L(s)=\frac{1}{\left|1-\boldsymbol{G}_{\mathrm{I}} \boldsymbol{G}_{\mathrm{II}} \boldsymbol{G}_{\mathrm{III}}(s)\right|}
$$

It can be seen that since $\left|1-\boldsymbol{G}_{\mathrm{I}} \boldsymbol{G}_{\mathrm{II}} \boldsymbol{G}_{\mathrm{III}}(s)\right|$ characterizes the distance of open-loop gain to the critical point $(1,0 \mathrm{j}), L(s)$ carries the information of stability margin, e.g., the larger the value of $L(s)$, the less the stability margin is.

\section{Interpretation of the main findings}

\section{1) Interpretation of the cause of different sensitivities of} stability to FFP location change

According to Fig. 10, the cause of different stability sensitivities to the change of FFP location can be overall interpreted like this: since the transfer-functions in the forwardpath exhibited by Type-I IV implementations (i.e., $\boldsymbol{G}_{\mathrm{I}, \text { Typel } \sim \mathrm{IV}}$ ) are different, their resulting closed-loop stability characteristics will be different. To further explain that Type-I is similar to Type-III while different from Type-II or -IV, the sensitivity functions of SOGI-PLL/FLL (i.e., $L_{\text {SOGI_PLL,FLL }}$ ) with different $k_{\text {Sogi }}$ and $\alpha_{P L L, F L L}$ will be analyzed, as shown in Fig. 11.

First, from Fig. 11 (a) it can be seen that the differences among Type-I IV implementations are small when $k_{\text {sogi }}$ is small. This is mainly attributed to the filtering effect of SOGIQSG, where a small gain generally makes the SOGI-QSG more 
selective. Thus, the effects of different models of $\boldsymbol{G}_{\mathrm{I} \text {, TypeI IV }}$ are greatly attenuated.

However, when the SOGI gain is increased, evident differences between Type-I/III and Type-II/IV present. This can be more clearly seen in Fig. 11 (b), where Type-I IV implementations are compared under two types of PLL/FLL bandwidths with a relatively high SOGI-gain.
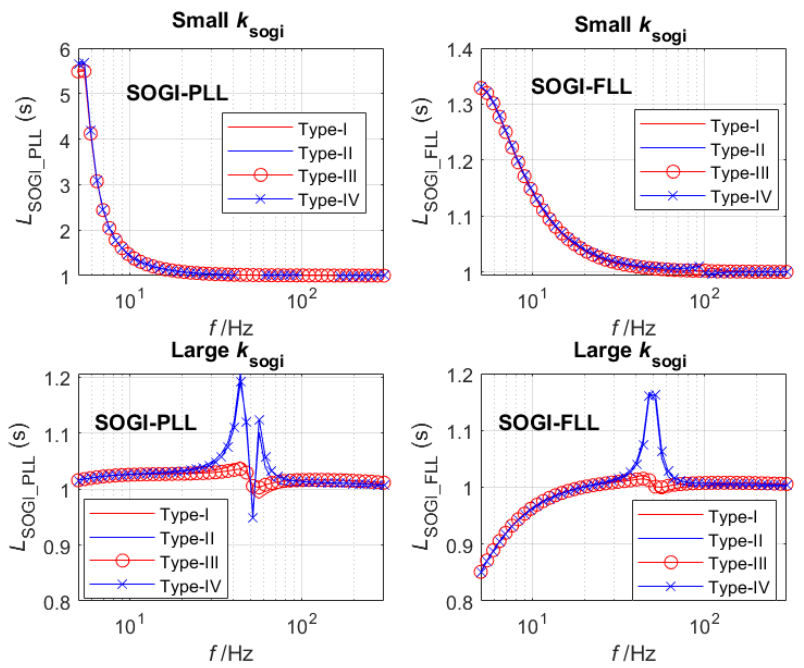

(a) under different SOGI gains
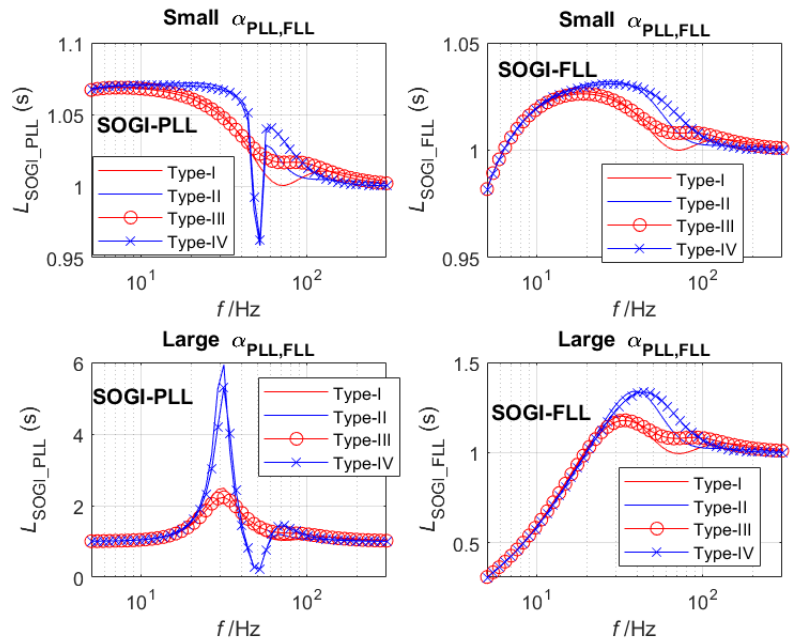

(b) under different PLL/FLL bandwidths

Fig. 11 Comparative analysis of SOGI-PLL/FLL using sensitivity functions. In (a), small: $k_{\text {sogi }}=0.2$, large: $k_{\text {sogi }}=10, \alpha_{\mathrm{PLL}, \mathrm{FLL}}=20 \mathrm{~Hz}$. In (b), small: $\alpha_{\mathrm{PLL}, \mathrm{FLL}}=10 \mathrm{~Hz}$; large: $\alpha_{\mathrm{PLL}, \mathrm{FLL}}=100 \mathrm{~Hz} ; \mathrm{k}_{\mathrm{sogi}}=2$.

\section{2) Interpretation of the better stability margin of Type-I/III than Type-II/IV}

As mentioned earlier, the sensitivity function also carries information about the system's stability margin. Based on this fact and by examining the characteristics of sensitivity functions given in Fig. 11, it can be seen that for both SOGIPLL and -FLL, the $L(\mathrm{~s})$ of Type-I/III overall shows fewer fluctuations than the Type-II/IV. Also, the $L(\mathrm{~s})$ of Type-I/III has an overall lower magnitude (aside from the negative-directional spike at $50 \mathrm{~Hz}$ ). Therefore, it can be concluded that the stability margin of Type-I/III is indeed better than Type-II/IV.
3) Interpretation of wider stability region of SOGI-FLL than SOGI-PLL

The wider stability region of SOGI-FLL concluded from the parametric stability analysis can be translated into the implication that SOGI-FLL is more robust than SOGI-PLL against parameter variations, i.e., the margin of SOGI-FLL is less sensitive to the parameter change. To confirm this interpretation, the change of $L(\mathrm{~s})$ under different parameters (i.e., $\left.k_{\text {sogi }}, \alpha_{P L L, F L L}\right)$ can be examined, where the results of Fig. 11 can still be utilized for this analysis.

First, from Fig. 11 (a) it can be seen that regardless of the FFP implementations, the change of $L_{\text {SOGI_FLL }}$ is much smaller than $L_{\text {SOGI_PLL }}$ when $k_{\text {sogi }}$ varies. Thus the SOGI-FLL is more robust against the variation of $k_{\text {sogi }}$ than SOGI-PLL. Next, by examining Fig. 11 (b) it can be further confirmed that SOGIFLL is still more robust than SOGI-PLL concerning the variation of $\alpha_{P L L, F L L}$. In particular, for SOGI-PLL, the change (increase) of $\alpha_{P L L, F L L}$ leads to the occurrence of a large spike in the sensitivity function, indicating its stability margin is of high sensitivity to this parameter's variation.

Overall, the above interpretations agree with the parametric stability analysis. However, it is worth reminding that since the above-applied models are greatly simplified for interpretations, they cannot be used for rigorous and quantitative stability analysis. In this regard, the parametric stability analysis method based on high-fidelity system models should be used.

\section{CONCLUSIONS}

This paper presents a parameter space-oriented stability analysis method aiming at a thorough discussion on the smallsignal stability of two types of widely applied synchronization units, i.e., SOGI-PLL and -FLL. The stability difference arising from different FFP implementations in SOGI-QSG is identified and evaluated using the parametric stability analysis method. The results from the presented analysis demonstrate how the FFP implementation can lead to significant impacts on the overall stability performance of SOGI-PLL/FLL. Therefore, the choice of the FFP implementation should be carefully considered, especially in applications concerned with stability. In this respect, the conclusions and analysis of this paper can serve as a useful reference. The detailed findings and conclusions are listed as follows:

1) Regardless of the frequency adaptive law (i.e., either PLL or FLL is used), Type-I IV FFP implementations can be classified into two groups based on the stability characteristics: Type-I/III is one group, while Type-II/IV is another group.

2) Regardless of the types of synchronization units used, the stability performance of Type-I/III is overall better than TypeII/IV. Therefore, this conclusion suggests that it is better to configure or implement the SOGI-QSG as Type-I/III to gain more benefits on stability.

3) Under each FFP implementation, SOGI-FLL exhibits a wider stability region than SOGI-PLL, thus showing a better stability performance than SOGI-PLL. 


\section{APPENDIX}

\section{A. Frequency response of SOGI-PLL/FLL for model validation}

This Appendix shows the calculated and measured frequency responses of SOGI-PLL/FLL to further justify why the applied truncation-order (i.e., $N=4$ ) is adequate for this study.

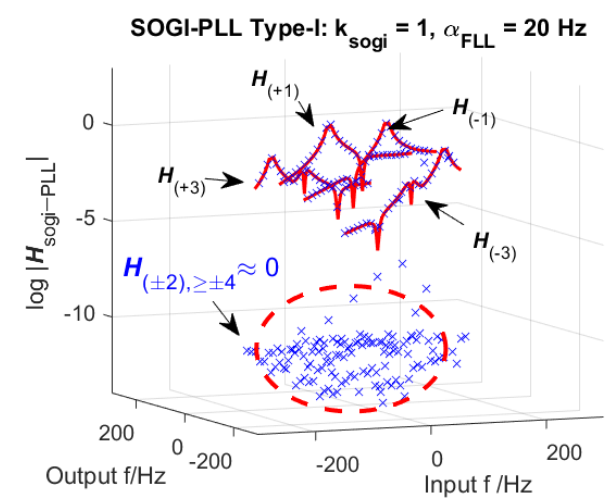

(a)

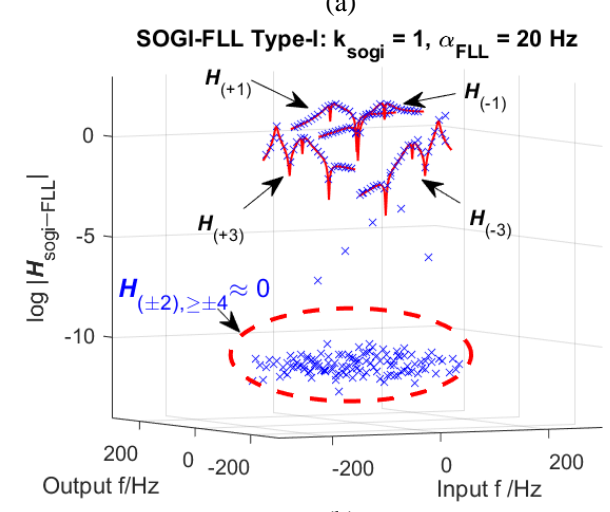

(b)

Fig.A1 Measured and calculated frequency responses of SOGI-PLL/FLL under Type-I (cross: measurements, line: analytic results)

To analyze the frequency response, the harmonic-transferfunction (HTF) [17] is used which can be derived from (3) as

$$
\mathcal{Y}=\underbrace{\left(\mathcal{C}(s \mathcal{I}-(\mathcal{A}-\mathcal{N}))^{-1} \mathcal{B}+\mathcal{D}\right)}_{\mathcal{H}(s)} \cdot \mathcal{U}
$$

where $\mathcal{H}(s)$ can be generally written as [21]:

$$
\mathcal{H}(s)=\left[\begin{array}{ccccc}
\ddots & \vdots & \vdots & \vdots & . \\
& H_{0}\left(s-\mathrm{j} \omega_{1}\right) & H_{-1}(s) & H_{-2}\left(s+\mathrm{j} \omega_{1}\right) & \\
H_{+1}\left(s-\mathrm{j} \omega_{1}\right) & H_{0}(s) & H_{-1}\left(s+\mathrm{j} \omega_{1}\right) & \\
H_{+2}\left(s-\mathrm{j} \omega_{1}\right) & H_{+1}(s) & H_{0}\left(s+\mathrm{j} \omega_{1}\right) & \\
\vdots & \vdots & \vdots & \ddots
\end{array}\right]
$$

Based on the HTF notation, HTFs of SOGI-PLL/FLL are respectively defined as:

$$
\boldsymbol{\delta}_{\mathrm{pll}}=\mathcal{H}_{\text {sogi-PLL }}(s) \cdot \mathcal{U}_{g} \text { and } \boldsymbol{x}_{\text {fll }}=\mathcal{H}_{\text {sogi-FLL }}(s) \cdot \mathcal{U}_{g}
$$

Then, frequency responses obtained from HTFs (i.e., analytic results) will be compared with those measured from simulations in PSCAD/EMTDC (i.e., measurements). Due to limited space, only the Type-I FFP implementation will be presented.

From Fig.A1 it can be obtained that, indeed, the measured frequency responses of SOGI-PLL/FLL with a harmonic order
$N \gg 4$ (see $\boldsymbol{H}_{(\geq 4)} \approx 0$ ) are very small and negligible, thus using $N=4$ is sufficiently precise for stability analysis.

In addition to the above main conclusion, these plots also demonstrate the merits of using the LTP method applied to model SOGI-PLL/FLL, where it can be seen that the frequency responses presented at $N= \pm 3$ can be accurately captured by components $\boldsymbol{H}_{( \pm 3)}$ from (A.3), whereas for the LTI modeling method, this precision is hard to achieve.

\section{B. Simplified frequency-domain modeling of the unified feedback systems}

\section{1) Input-output models of Block-I}

Transforming the Type-I IV linearized models in Fig. 9 (b) into the frequency-domain, yield the following equations:

$$
\begin{gathered}
{\left[\begin{array}{l}
\Delta u_{\omega}(s-\mathrm{j} \omega) \\
\Delta u_{\omega}(s+\mathrm{j} \omega)
\end{array}\right]=\underbrace{\left[\boldsymbol{G}_{\mathrm{I}, \text { Type-I }}(s)\right]_{2 \times 1}}_{\frac{\mathrm{j} U_{0}}{\omega_{1}}\left[\begin{array}{ll}
-1 & 1
\end{array}\right]^{T} \Delta \omega(s)}} \\
{\left[\boldsymbol{G}_{\mathrm{I}, \text { Type-II }}(s)\right]_{2 \times 1}=\left[-\frac{\mathrm{j} U_{0}}{2 \omega_{1}}-\frac{U_{0}}{2\left(s-\mathrm{j} \omega_{1}\right)} \frac{\mathrm{j} U_{0}}{2 \omega_{1}}-\frac{U_{0}}{2\left(s+\mathrm{j} \omega_{1}\right)}\right]^{T}} \\
{\left[\boldsymbol{G}_{\mathrm{I}, \text { Type-III }}(s)\right]_{2 \times 1}=\left[-\frac{\mathrm{j} U_{0}}{2 \omega_{1}}+U_{0} \frac{s-\mathrm{j} \omega_{1}}{2 \omega_{1}^{2}} \frac{\mathrm{j} U_{0}}{2 \omega_{1}}+U_{0} \frac{s+\mathrm{j} \omega_{1}}{2 \omega_{1}^{2}}\right]^{T}} \\
{\left[\boldsymbol{G}_{\mathrm{I}, \text { TypeIV }}(s)\right]_{2 \times 1}=\frac{U_{0}}{2}\left[\frac{\left(s-\mathrm{j} \omega_{1}\right)^{2}-\omega_{1}^{2}}{\left(s-\mathrm{j} \omega_{1}\right) \omega_{1}^{2}} \frac{\left(s+\mathrm{j} \omega_{1}\right)^{2}-\omega_{1}^{2}}{\left(s+\mathrm{j} \omega_{1}\right) \omega_{1}^{2}}\right]^{T}}
\end{gathered}
$$

\section{2) Input-output model of Block-II}

Based on the control block of Fig. 9 (a), the input-output model of SOGI-QSG can be obtained:

$$
\left[\begin{array}{c}
\Delta u_{g \alpha}(s) \\
\Delta u_{\mathrm{g} \beta}(s)
\end{array}\right]=\left[\begin{array}{c}
G_{\alpha_{-} \mathrm{II}} \\
G_{\beta \_} \mathrm{II}
\end{array}\right] \Delta u_{\omega}(s)
$$

where

$$
G_{\mathrm{II}, \alpha}=\frac{s \omega_{1}}{s^{2}+s \omega_{1} k_{\mathrm{sogi}}+\omega_{1}^{2}}, G_{\mathrm{II}, \beta}=\frac{\omega_{1}}{s} G_{\mathrm{II}, \alpha}
$$

3) Input-output models of Block-III

For FLL, the model is:

$$
\Delta \omega_{F L L}(s)=\underbrace{\frac{U_{0}}{2 i} \frac{\alpha_{F L L} k_{\mathrm{sog}} \omega_{1}}{s U_{0}^{2}}[1}_{G_{\mathrm{II}, \mathrm{FL}}(s)}-1]\left[\begin{array}{c}
\Delta u_{\mathrm{g} \alpha}(s-\mathrm{j} \omega) \\
\Delta u_{\mathrm{g} \alpha}(s+\mathrm{j} \omega)
\end{array}\right]
$$

The connecting matrix with Block-II is:

$$
\left[\begin{array}{c}
\Delta u_{\mathrm{g} \alpha}(s-\mathrm{j} \omega) \\
\Delta u_{\mathrm{g} \alpha}(s+\mathrm{j} \omega)
\end{array}\right]=\underbrace{\left[\begin{array}{cc}
G_{\alpha_{-} \mathrm{II}}(s-\mathrm{j} \omega) & 0 \\
0 & G_{\alpha_{-} \mathrm{II}}(s+\mathrm{j} \omega)
\end{array}\right]}_{\left[\boldsymbol{G}_{\mathrm{II},}\right]_{2 \times 2}}\left[\begin{array}{l}
\Delta u_{\omega}(s-\mathrm{j} \omega) \\
\Delta u_{\omega}(s+\mathrm{j} \omega)
\end{array}\right]
$$

For the PLL, the model is:

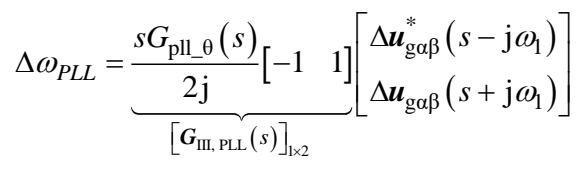

where $\Delta \boldsymbol{u}_{\mathrm{g} \alpha \beta}(s)=\Delta u_{\mathrm{g} \alpha}(s)+\mathrm{j} \Delta u_{\mathrm{g} \beta}(s), \Delta \boldsymbol{u}_{\mathrm{g} \alpha \beta}^{*}=\Delta u_{\mathrm{g} \alpha}(s)-\mathrm{j} \Delta u_{\mathrm{g} \beta}(s)$. 
The connecting matrix with Block-II is:

$$
\left[\begin{array}{c}
\Delta \boldsymbol{u}_{\mathrm{g} \alpha \beta}^{*}\left(s-\mathrm{j} \omega_{1}\right) \\
\Delta \boldsymbol{u}_{\mathrm{g} \alpha \beta}\left(s+\mathrm{j} \omega_{1}\right)
\end{array}\right]=\underbrace{\left[\begin{array}{cc}
G_{\alpha \beta_{-} \text {II }}^{*}\left(s-\mathrm{j} \omega_{1}\right) & 0 \\
0 & G_{\alpha \beta_{-} \mathrm{II}}\left(s+\mathrm{j} \omega_{1}\right)
\end{array}\right]}_{\left[\boldsymbol{G}_{\mathrm{II}, \alpha \beta}\right]_{2 \times 2}}\left[\begin{array}{c}
\Delta u_{\omega}(s-\mathrm{j} \omega) \\
\Delta u_{\omega}(s+\mathrm{j} \omega)
\end{array}\right]
$$

where $G_{\mathrm{II}, \alpha \beta}(s)=G_{\mathrm{II}, \alpha}+\mathrm{j} G_{\mathrm{II}, \beta}$, and $G_{\mathrm{II}, \alpha \beta}^{*}(s)=G_{\mathrm{II}, \alpha}-\mathrm{j} G_{\mathrm{II}, \beta}$.

\section{REFERENCES}

[1] R. Teodorescu, M. Liserre, and P. Rodriguez, Grid Converters for Photovoltaic and Wind Power Systems. New York, NY, USA: Wiley, 2011

[2] F. D. Freijedo, A. G. Yepes, Ó. López, A. Vidal and J. Doval-Gandoy, "Three-Phase PLLs With Fast Postfault Retracking and Steady-State Rejection of Voltage Unbalance and Harmonics by Means of Lead Compensation," in IEEE Transactions on Power Electronics, vol. 26, no. 1, pp. 85-97, Jan. 2011

[3] P. Rodríguez, R. Teodorescu, I. Candela, A. V. Timbus, M. Liserre and F. Blaabjerg, "New positive-sequence voltage detector for grid synchronization of power converters under faulty grid conditions," 2006 37th IEEE Power Electronics Specialists Conference, Jeju, 2006, pp. 1-7

[4] M. Ciobotaru, R. Teodorescu and F. Blaabjerg, "A new single-phase PLL structure based on second order generalized integrator," 2006 37th IEEE Power Electronics Specialists Conference, Jeju, 2006, pp. 1-6.

[5] Xiaoming Yuan, W. Merk, H. Stemmler and J. Allmeling, "Stationaryframe generalized integrators for current control of active power filters with zero steady-state error for current harmonics of concern under unbalanced and distorted operating conditions," in IEEE Transactions on Industry Applications, vol. 38, no. 2, pp. 523-532, March-April 2002.

[6] D. N. Zmood and D. G. Holmes, "Stationary frame current regulation of PWM inverters with zero steady state error," 30th Annual IEEE Power Electronics Specialists Conference. Record. (Cat. No.99CH36321), Charleston, SC, USA, 1999, pp. 1185-1190 vol.2.

[7] J. Matas, H. Martín, J. de la Hoz, A. Abusorrah, Y. A. Al-Turki and M. AlHindawi, "A Family of Gradient Descent Grid Frequency Estimators for the SOGI Filter," in IEEE Transactions on Power Electronics, vol. 33, no. 7, pp. 5796-5810, July 2018.

[8] S. Golestan, J. M. Guerrero, A. Abusorrah, M. M. Al-Hindawi and Y. AlTurki, "An Adaptive Quadrature Signal Generation-Based Single-Phase Phase-Locked Loop for Grid-Connected Applications," in IEEE Transactions on Industrial Electronics, vol. 64, no. 4, pp. 2848-2854, April 2017.

[9] F. Xiao, L. Dong, L. Li and X. Liao, "A Frequency-Fixed SOGI-Based PLL for Single-Phase Grid-Connected Converters," in IEEE Transactions on Power Electronics, vol. 32, no. 3, pp. 1713-1719, March 2017.

[10] P. Rodriguez, A. Luna, I. Candela, R. Teodorescu and F. Blaabjerg, "Grid synchronization of power converters using multiple second order generalized integrators," 2008 34th Annual Conference of IEEE Industrial Electronics, Orlando, FL, 2008, pp. 755-760.

[11] D. B. Rao and Sun-Yuan Kung, "Adaptive notch filtering for the retrieval of sinusoids in noise," in IEEE Transactions on Acoustics, Speech, and Signal Processing, vol. 32, no. 4, pp. 791-802, August 1984.

[12] S. Golestan, J. M. Guerrero, F. Musavi and J. C. Vasquez, "Single-Phase Frequency-Locked Loops: A Comprehensive Review," in IEEE Transactions on Power Electronics, vol. 34, no. 12, pp. 11791-11812, Dec. 2019.

[13] X. He, H. Geng and G. Yang, "Reinvestigation of Single-Phase FLLs," in IEEE Access, vol. 7, pp. 13178-13188, 2019.

[14] P. Rodriguez, A. Luna, M. Ciobotaru, R. Teodorescu and F. Blaabjerg, "Advanced Grid Synchronization System for Power Converters under Unbalanced and Distorted Operating Conditions," IECON 2006 - 32nd Annual Conference on IEEE Industrial Electronics, Paris, 2006, pp. 51735178

[15] P. Rodríguez, A. Luna, I. Candela, R. Mujal, R. Teodorescu and F. Blaabjerg, "Multiresonant Frequency-Locked Loop for Grid Synchronization of Power Converters Under Distorted Grid Conditions," in IEEE Transactions on Industrial Electronics, vol. 58, no. 1, pp. 127-138, Jan. 2011.

[16] C. M. Hackl and M. Landerer, "Modified Second-Order Generalized Integrators With Modified Frequency Locked Loop for Fast Harmonics
Estimation of Distorted Single-Phase Signals," in IEEE Transactions on Power Electronics, vol. 35, no. 3, pp. 3298-3309, March 2020.

[17] N. M. Wereley, "Analysis and control of linear periodically time varying systems," Ph.D. dissertation, Department of Aeronautics and Astronautics, Massachusetts Institute of Technology, 1990.

[18] S. Golestan, J. M. Guerrero and J. C. Vasquez, "Modeling and Stability Assessment of Single-Phase Grid Synchronization Techniques: Linear Time-Periodic Versus Linear Time-Invariant Frameworks," in IEEE Transactions on Power Electronics, vol. 34, no. 1, pp. 20-27, Jan. 2019.

[19] V. Salis, A. Costabeber, S. M. Cox and P. Zanchetta, "Stability Assessment of Power-Converter-Based AC systems by LTP Theory: Eigenvalue Analysis and Harmonic Impedance Estimation," in IEEE Journal of Emerging and Selected Topics in Power Electronics, vol. 5, no. 4, pp. 1513-1525, Dec. 2017.

[20] Lazarus, Arnaud, and Olivier Thomas. "A harmonic-based method for computing the stability of periodic solutions of dynamical systems." Comptes Rendus Mécanique 338, no. 9 (2010): 510-517.

[21] Möllerstedt, Erik. "Dynamic analysis of harmonics in electrical systems." $\mathrm{PhD}$ diss., Department of Automatic Control, Lund Institute of Technology, 2000.

[22] B. Wen, D. Boroyevich, R. Burgos, P. Mattavelli and Z. Shen, "SmallSignal Stability Analysis of Three-Phase AC Systems in the Presence of Constant Power Loads Based on Measured d-q Frame Impedances," in IEEE Transactions on Power Electronics, vol. 30, no. 10, pp. 5952-5963, Oct. 2015.

[23] C. Zhang, M. Molinas, S. Foyen, J. A. Suul and T. Isobe, "An Integrated Method for Generating VSCs' Periodical Steady-state Conditions and HSS-based Impedance Model," in IEEE Transactions on Power Delivery.

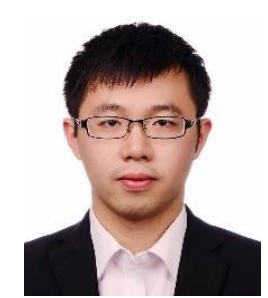

Chen Zhang received the B.Eng. degree from the China University of Mining and Technology, China, and the Ph.D. from Shanghai Jiao Tong University, China, in 2011 and 2018 respectively. He was a Postdoctoral Research Fellow at the Department of Engineering Cybernetics of NTNU, from March 2018 to October 2020. Currently, he is a postdoc with the Department of Electrical Engineering, Technical University of Denmark, Lyngby, Denmark. His research interest is modeling and stability analysis of VSC-based energy conversion systems, where the aim is to reveal the fundamental dynamics and stability mechanisms of renewable energies with VSCs as the grid interface.

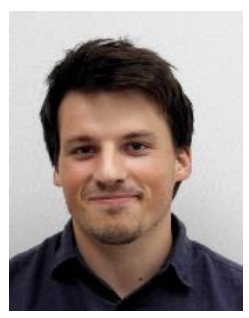

Sjur Føyen (M'18) received the Master's degree in electric power engineering from the Norwegian University of Science and Technology, Trondheim, Norway, in 2018. Currently, he is pursuing his doctoral degree in power electronics synchronization and stability. His research interests include synchronization, time-frequency analysis, and stability of power (electronics) systems.

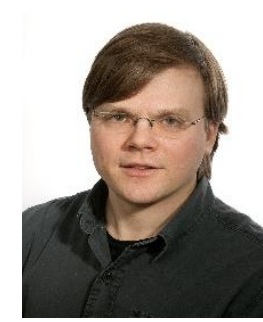

Jon Are Suul (M'11) received the M.Sc. degree in energy and environmental engineering and the Ph.D. degree in electric power engineering from the Norwegian University of Science and Technology (NTNU), Trondheim, Norway, in 2006 and 2012, respectively. From 2006 he was with SINTEF Energy Research, Trondheim, where he was working with simulation of power electronic converters and marine propulsion systems until starting his $\mathrm{PhD}$ studies. From 2012, he resumed a position as a Research Scientist at SINTEF Energy Research, first in part-time position while also working as a part-time postdoctoral researcher at the Department of Electric Power Engineering of NTNU until 2016. Since August 2017, he is serving as an Adjunct Associate Professor at the Department of Engineering Cybernetic of NTNU. His research interests are mainly related to modelling, analysis and control of power electronic converters in HVDC transmission systems, renewable energy applications and electrification of transport. Dr. Suul is an Associate Editor for the IEEE JOURNAL OF EMERGING AND SELECTED TOPICS IN POWER ELECTRONICS 
Marta Molinas (M'94) received the Diploma degree in electromechanical

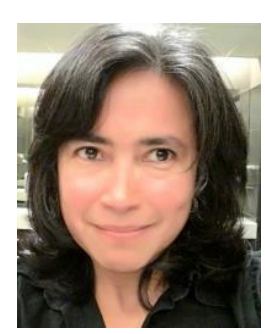
engineering from the National University of Asuncion, Asuncion, Paraguay, in 1992; the Master of Engineering degree from Ryukyu University, Japan, in 1997; and the Doctor of Engineering degree from the Tokyo Institute of Technology, Tokyo, Japan, in 2000. She was a Guest Researcher with the University of Padova, Padova, Italy, during 1998. From 2004 to 2007, she was a Postdoctoral Researcher with the Norwegian University of Science and Technology (NTNU) and from 20082014 she has been professor at the Department of Electric Power Engineering at the same university. She is currently Professor at the Department of Engineering Cybernetics, NTNU. Her research interests include stability of power electronics systems, harmonics, instantaneous frequency, and non-stationary signals from the human and the machine. She is Associate Editor for the IEEE Journal JESTPE, IEEE PELS Transactions and Editor of the IEEE Transactions on Energy Conversion. Dr. Molinas has been an AdCom Member of the IEEE Power Electronics Society from 2009 to 2011. 